\title{
Finding 47:23 in the Baby Monster
}

\author{
John N. Bray, Richard A. Parker and Robert A. Wilson
}

\begin{abstract}
In this paper we describe methods for finding very small maximal subgroups of very large groups, with particular application to the subgroup 47:23 of the Baby Monster. This example is completely intractable by standard or naïve methods. The example of finding 31:15 inside the Thompson group Th is also discussed as a test case.
\end{abstract}

\section{Introduction}

When computing in finite groups, it is often useful to know how to find generating sets for various subgroups, especially maximal ones, of a group $G$, in terms of the standard generators of $G$. Usually this means either as words or as straight-line programs: here we use 'word' loosely to cover both concepts. The WwW-ATLAS [11] now includes such words for maximal subgroups of many groups. Some of the small maximal subgroups, however, pose particular challenges.

One place where we thought at one time the Www-ATLAS would have a permanent gap was for a straight-line program to find generators for the maximal subgroup 47:23 of the Baby Monster $\mathbb{B}$, owing to pessimistic forecasts for how long such a search would take. However, in 2003 we managed to fill in this gap by devising a new and more subtle search strategy. In this paper we describe this method, in the hope that it may be of use in other similar searches.

There are various reasons why our main problem is particularly difficult, such as $\mathbb{B}$ being large (order approximately $4.15478 \times 10^{33}$ ) and that we are constrained to compute in a large representation (degree 4370 over $\mathbb{F}_{2}$ ). Moreover, the non-trivial elements of 47:23 have very small centralisers in $\mathbb{B}$ (order 46 or 47 ). And every non-trivial proper subgroup of 47:23 is cyclic of prime order, so we cannot even search in another subgroup of $\mathbb{B}$ to give us a useful contribution towards generating 47:23.

We first tested the new strategy by using it to obtain words for generators of 31:15 in terms of standard generators of the Thompson group Th. Since Th has a considerably smaller representation than $\mathbb{B}$ (degree 248 as opposed to 4370 over $\mathbb{F}_{2}$ ), this provided an ideal 'dry run' opportunity to try out various methods. It is worth pointing out that finding $31: 15<\mathrm{Th}$ is amenable to other techniques, and these include a brute force direct search, which we have also done. One of the main reasons for this is that 15 , unlike 23 , is not prime. The method Wilson [10] employs to find 31:15 < Th relies on 15 not being prime.

For the purposes of this paper, we shall assume that we have a standard copy of $\mathbb{B}$, on its standard generators, given as a subgroup of $\mathrm{GL}_{4370}(2)$ (the one whose www-ATLAS identifier is BG1-f2r4370B0). Likewise, we assume that we have a standard copy of Th as a subgroup of $\mathrm{GL}_{248}(2)$.

The calculations described in this paper can now be performed easily in Magma $[\mathbf{1}, \mathbf{3}]$. Originally, we used the C-Meataxe $[\mathbf{8}, \mathbf{9}]$ for the computationally intensive parts of the Baby Monster calculations (mainly calculating the 'fingerprints' in Step 1).

Received 1 June 2015; revised 20 November 2015.

2010 Mathematics Subject Classification 20B40 (primary), 20H99 (secondary). 


\section{A standard method}

Of the various methods the authors of the WWW-ATLAS $[\mathbf{1 1}]$ have commonly employed to find words for subgroups of a group $G=\langle a, b\rangle$, the least implausible method in the present instance (the case when $G=\mathbb{B}$ and the subgroup is $47: 23$ ) is to search at random through subgroups generated by two elements of order 23 .

Given fixed elements $x, y \in G$ (an arbitrary finite group), and elements $x^{\prime}$ and $y^{\prime}$ such that $x$ and $x^{\prime}$ are $G$-conjugate and $y$ and $y^{\prime}$ are $G$-conjugate, the probability that the pair $\left(x^{\prime}, y^{\prime}\right)$ is $G$-conjugate to $(x, y)$ is

$$
\frac{\left|\mathrm{C}_{G}(x)\right| \cdot\left|\mathrm{C}_{G}(y)\right|}{|G| \cdot\left|\mathrm{C}_{G}(\langle x, y\rangle)\right|},
$$

with $\left|\mathrm{C}_{G}(\langle x, y\rangle)\right|=1$ for the cases that interest us here.

Suppose we choose to generate $47: 23$ by two elements $x$ and $y$ of order 23 that are $\mathbb{B}$ conjugate. Since $\mathbb{B}$ has 242 conjugacy classes of (23A,23A) pairs that generate $47: 23$, the probability of success at each attempt is

$$
\frac{46 \cdot 46 \cdot 242}{|\mathbb{B}|}=\frac{253}{2052757648827285667577856000000} \approx \frac{1}{8.114 \times 10^{27}} .
$$

A test for success could be that $[[x, y],[[x, y], y]]=1$ and $[x, y]$ has order 47 . Trying to generate $47: 23$ by two non-conjugate (in $\mathbb{B}$ ) elements of order 23 gives us the same probability of success as above, and the same test for success still works. We reiterate a point made by Linton [7]: the thing we must test quickly is failure.

For the $(23 \mathrm{~A}, 23 \mathrm{~A})$ case, we know that for $1 \leqslant i \leqslant 22$, the order of $x y^{i}$ should be 23 . So we would first test, using a carefully written 'Monte Carlo order oracle', that $x y$ does not have order 23. If $x y$ does (or might) have order 23, we then perform the same test for $x y^{2}$ and (if $x y^{2}$ has order 23) for $x y^{3}$. At some point, we decide we are not gaining much by performing more of these failure tests, and perform the success test instead. (For the (23A, 23B) case, the elements $x y^{i}$ can have orders 23 or 47 , and overall the test would take a bit more than twice as long on average.)

Suppose, optimistically, that we can check $10^{12}$ cases a year (which is more than 30000 per second). Then we would still expect the search to take more than $8 \times 10^{15}$ years to complete, which compares unfavourably to the current age of the universe, which is thought to be about $1.38 \times 10^{10}$ years.

It is worth remarking that this method is essentially a black-box method, even though some of the tests are substantially speeded up by using fast Monte Carlo order oracles, or pseudoorder oracles, which depend on the representation for their implementation.

\section{A new method}

Our method is actually a method for finding the normaliser of a cyclic subgroup (or more generally, any small subgroup) inside a group $H$ that lies in an ambient general linear group or symmetric group $G$. We suppose that $H$ is generated by a relatively small conjugacy class $X$ of elements of $H$. Let $y$ be a generator for the cyclic group we wish to normalise.

Step 1. Locate pairs $\left(x_{1}, y\right)$ and $\left(x_{2}, y^{k}\right)$ that are $H$-conjugate, with $x_{i} \in X$ and $k$ a suitable integer, and $\left\langle x_{1}, y\right\rangle=H$.

Step 2. Use standard computational methods to find all elements $g \in G$ that conjugate $\left(x_{1}, y\right)$ to $\left(x_{2}, y^{k}\right)$.

Step 3. For one such $g \in H$, write $g$ as a word in the generators of $H$.

Step 1 is accomplished by a fingerprinting (or hashing) technique, described in more detail in $\S 5.1$, to detect coincidences in a large population. In this context, a fingerprint of a pair $(a, b)$ of group elements is a suitable collection of conjugacy-invariant data, for example a list of 
orders of certain elements, such as $a b, a b^{2},[a, b], \ldots$ A similar fingerprint for representations of group algebras is described in [8]. The essential ingredient of our method is to use the 'birthday paradox' to find coincidences quickly. Suppose that $x_{1}, \ldots, x_{m}$ are chosen from a set of size $d$. Then the probability of at least one coincidence between an $x_{i}$ and an $x_{j}$ exceeds $50 \%$ if $m^{2}>2\left(\log _{\mathrm{e}} 2\right) d$, and it exceeds $90 \%$ if $m^{2}>2\left(\log _{\mathrm{e}} 10\right) d$.

Step 2 is achieved by a 'standard basis' algorithm, such as can be found in $[\mathbf{5}, \S 7.5 .3]$ (see also $[8])$. The 'standard basis' is not in general unique, and $g$ is only determined up to multiplication by of the centraliser of the representation. However, in our examples, in fact the standard basis element is unique.

Step 3 is a 'constructive membership' problem, for which a number of methods, such as Ryba's algorithm [6], are available. However, Ryba's algorithm tends to produce quite long words, and we prefer a method which produces shorter words.

Our approach to finding a word for $g \in H$ (where $H$ is a finite group) in terms of the generators of $H$ is straightforward, even naïve: we use a process of successive approximation. So let

$$
H=H_{0}>H_{1}>H_{2}>\ldots>H_{r}=1
$$

be a chain of subgroups of $H$, where the generators of $H_{i}$ are given as words in the generators of $H_{i-1}$, and thus ultimately as words in the generators of $H$. The basic idea is to run through the right coset representatives $h_{0}$ of $H_{1}$ in $H_{0}$ until we find one for which $H_{1} h_{0}=H_{1} g$. Thus $g h_{0}^{-1} \in H_{1}$ and we recurse the problem into $H_{1}$, next finding $h_{1} \in H_{1}$ such that $H_{2} g h_{0}^{-1}=$ $H_{2} h_{1}$, giving $g h_{0}^{-1} h_{1}^{-1} \in H_{2}$. Continuing in this manner, we eventually find elements $h_{0} \in$ $H_{0}, h_{1} \in H_{1}, \ldots, h_{r-1} \in H_{r-1}$ such that $g=h_{r-1} \ldots h_{1} h_{0}$. Of course, each $h_{i}$ is written as a word in the generators of $H_{i}$.

Some of the indices we encounter below are somewhat too large to make such a naïve approach feasible, and again, the 'birthday paradox' comes to our rescue. Instead of searching through all the cosets $H_{1} h_{0}$ until we find $H_{1} g$, we make a collection of cosets $H_{1} x_{i}$, and another collection $H_{1} g y_{j}$, and look for coincidences between these two sets.

Suppose that $x_{1}, \ldots, x_{m}$ and $y_{1}, \ldots, y_{n}$ are chosen from a set of size $d$. Then the probability of at least one coincidence between an $x_{i}$ and a $y_{j}$ exceeds $50 \%$ if $m n>\left(\log _{\mathrm{e}} 2\right) d$, and it exceeds $90 \%$ if $m n>\left(\log _{\mathrm{e}} 10\right) d$. Therefore if $H_{1}$ has index $d$ in $H_{0}$ and we make equal numbers of 'forward' and 'back' cosets (that is, cosets $H_{1} x_{i}$ and $H_{1} g y_{j}$ ), we need to make about $2 \sqrt{\left(\log _{\mathrm{e}} 2\right) d}$ cosets for the probability of coincidence to be at least $50 \%$, and probably somewhat more than that to account for insufficient randomness in our production of cosets.

\section{Application to the Thompson group}

We first describe how one can use this new method to find a copy of 31:15, the normaliser of a subgroup of order 31, in Th. We use the embedding $\mathrm{Th} \leqslant \mathrm{GL}_{248}(2)$. For this experimental case, we did try out Ryba's algorithm, regarding the involution centraliser $2_{+}^{1+8} \cdot \mathrm{A}_{9}$ as the base case. The result of this is given as the program ThG1-max15W1 in the WwW-ATLAS. Thereafter, we quickly produced another version (ThG1-max15W2), with shorter words, using other methods for constructive membership testing, as described below.

We pick an element $y$ of order 31. The quadratic residues modulo 31 are the powers of 7 , that is:

$$
1,7,18,2,14,5,4,28,10,8,25,20,16,19,9 .
$$

The conjugacy class $X$ is taken to be Class 2A, which is the class of 976841775 involutions.

Step 1. For the first step, we must fingerprint pairs $(x, y)$ with $x \in X$. It is not quite true that all such pairs must generate Th, for among the 976841775 involutions, one calculates that $3 \times 7740$ of them generate with $y$ a copy of $2^{5} . L_{5}(2)$ (for a probability of $16 / 700245$ ) and 
that $3 \times 31$ of them generate $2^{5}: 31$ (for a probability of $1 / 10503675$ ). So the probability of a proper subgroup is 241/10503675, which is approximately 1 in 43584 . This is small enough that it does not materially affect the calculations.

A convenient fingerprint is the list of orders of $x y^{7^{k}}$, for $0 \leqslant k \leqslant 14$. This was chosen because 7 is an element of multiplicative order 15 modulo 31. Indeed, the 8 elements of order 15 modulo 31 are just $7^{k}$ for $k$ coprime to 15 . Replacing $y$ by $y^{7}$ then just has the effect of cycling the 15 orders in this fingerprint. Thus a relevant coincidence is detected by finding two fingerprints which differ only by one of the 8 rotations of order 15 . We expect around 2 million different cyclically reduced fingerprints, and therefore expect to find a coincidence after inspecting a few thousand of them.

Step 2. Once we have found a candidate coincidence in this way, Step 2 allows us both to prove that it is a genuine coincidence, and to find an element $g$ of $\mathrm{GL}_{248}(2)$ which conjugates the relevant pairs: $\left(x_{1}, y\right)$ to $\left(x_{2}, y^{7^{k}}\right)$. Now the fact that this representation of Th is absolutely irreducible, over the field of two elements, implies that there is a unique such element $g$, and in particular, $g \in$ Th. Of course, we want $g$ to have order 15 , which means that the coincidence we use must have $k$ prime to 15 . Otherwise, $g$ has smaller order. (An alternative is to find one element of order 3 and one of order 5 , rather than an element of order 15.)

Step 3. The final step is now to find a word for $g$ in terms of the standard generators for Th. One method for doing this is Ryba's algorithm, which is effective in this case, but gives words which are perhaps longer than we might hope for. Since this is a one-off calculation, it is worth adopting a rather more labour-intensive method in order to obtain shorter words, as described in $\S 3$.

We pick a subgroup chain (of length 2),

$$
\mathrm{Th}>2_{+}^{1+8 \cdot} \cdot \mathrm{A}_{9}>1,
$$

with indices 976841775 and 92897280 . Our strategy is, essentially, first to identify which coset of the subgroup $2_{+}^{1+8} \cdot A_{9}$ the element $g$ lies in, and then to identify which element of that coset it is. In both steps, we use the 'birthday problem' to square root the size of the search.

Now in the given representation of $\mathrm{Th}$, the subgroup $2_{+}^{1+8} \cdot \mathrm{A}_{9}$ fixes a unique non-zero vector $v$, and we may use the orbit of $v$ under Th to represent the cosets of $2_{+}^{1+8} \cdot \mathrm{A}_{9}$ therein. The method is to make around 100000 images of $v$ and $v^{g}$ under known elements of Th, and search for coincidences. Any such coincidence gives rise to an element $w$ of Th such that $g w$ fixes $v$, and therefore $g w$ lies in the given subgroup which is the stabiliser of $v$.

Within the subgroup, we first chopped the representation, and chose a suitable subquotient representing the group $2^{8} \cdot A_{9}$. In fact, we were able to choose a subspace of dimension 29 , namely the third socle. The dimension was small enough that we could compute enough group elements to locate a coincidence directly. Hence we obtained a word for $g w$, which now only had to be possibly adjusted by the central involution (of $2_{+}^{1+8} \cdot A_{9}$ ) in order to complete the task.

\section{Application to the Baby Monster}

We apply the same basic method outlined in $\S 3$ to the problem of finding 47:23 in $\mathbb{B}$ as follows. We assume that the 47 -subgroup we wish to normalise is generated by $y$, and that $\mathbb{B}$ sits in some 'universal' group $G$, which in our case is $G=\mathrm{GL}_{4370}(2)$. Specifically, we let $\mathbb{B}=\langle a, b\rangle \leqslant$ $\mathrm{GL}_{4370}(2)$, where $a$ and $b$ are standard generators of $\mathbb{B}$, that is $a$ is in Class $2 \mathrm{C}, b$ is in Class $3 \mathrm{~A}, a b$ has order 55 and $a b a b a b a b^{2} a b a b^{2} a b^{2}$ has order 23. Our standard subgroup of order 47 is then taken to be $y=a b a b a b^{2} a b a b a b a b^{2} a b a b^{2}$, which we shall deem to lie in Class 47A. The normalising element $g$ that we are looking for conjugates $y$ to $y^{k}$ for some $k \neq 1$ which is a quadratic residue modulo 47 . The quadratic residues modulo 47 are the powers of 2 . We let 
$x$ run through the smallest non-trivial conjugacy class in $\mathbb{B}$, so that the number of possibilities for $(x, y)$ is as small as possible. This is the class of transpositions (Class 2A in the AtLAs [4, page 208]).

\subsection{Step 1}

Any $(2 \mathrm{~A}, 47 \mathrm{~A})$ pair of elements must generate the whole of $\mathbb{B}$, since the only group $H$ such that $\mathbb{B}>H>\langle y\rangle \cong 47$ is $H=\mathrm{N}_{\mathbb{B}}(\langle y\rangle) \cong 47: 23$. Now $\mathbb{B}$ has 13571955000 transpositions, and all subgroups of order 47 are self-centralising, so the probability that any (2A, 47A) pair is conjugate in $\mathbb{B}$ to a given one is:

$$
\frac{47}{13571955000}=\frac{1}{288765000} .
$$

Naturally, we do not wish to collect fingerprints of the 288765000 distinct (2A,47A) pairs (and the same number for the (2A,47B) pairs) in order to define a pair of $(2 \mathrm{~A}, 47 \mathrm{~A})$ standard generators of $\mathbb{B}$. Nor would we wish to trawl through an expected 288765000 transpositions $x_{2}$ until we found one such that $\left(x_{2}, y^{2^{k}}\right)$ is conjugate to our favourite $(2 \mathrm{~A}, 47 \mathrm{~A})$ pair $\left(x_{1}, y\right)$.

We have already observed that we wish to conjugate $y$ to some non-trivial power $y^{m}$, where necessarily $m$ is a square modulo 47 , and without loss of generality $1<m<47$. It is convenient for later computations to arrange the possible values of $m$ as successive powers of 2 , and so the set $S$ of allowed values of $m$ modulo 47 is:

$$
S:=\{2,4,8,16,32,17,34,21,42,37,27,7,14,28,9,18,36,25,3,6,12,24\} .
$$

The fingerprint of a pair $(x, y)$ is the list of the orders of the 23 elements $x y^{2^{k}}$, as $k$ runs from 0 to 22. We used a Monte Carlo order oracle, which has a very small chance of giving a proper divisor of the actual element order. In principle, this could cause us to miss a genuine coincidence, or to flag up a false coincidence. The former is not material, while the latter is detected at the next stage, so can be thrown away and a new coincidence tested.

The probability that $\left(x_{1}, y\right)$ is conjugate to $\left(x_{2}, y^{m}\right)$ for one of 22 values of $m \in S$ is $p=11 / 144382500$, which is still uncomfortably low. But we are not looking for a particular fingerprint, we are just looking for coincidences between fingerprints. That is, we wish to make sufficient transpositions $x_{1}, x_{2}, \ldots, x_{r}$ such that $\left(x_{i}, y\right)$ and $\left(x_{j}, y^{m}\right)$ are conjugate for some $m \in S$. As described in $\S 3$, this is a form of the 'birthday problem', and somewhere around $r=10000$ gives us a reasonably good chance of finding a coincidence. Note, however, that we are not interested in coincidences with $m=1$. In the end, we considered slightly fewer than 10000 fingerprints, and found exactly one coincidence of the required type. It transpires that the coincidence we found was between pairs of the form $\left(x_{1}, y\right)$ and $\left(x_{2}, y^{16}\right)$.

\subsection{Step 2}

Since $\left(x_{1}, y\right)$ and $\left(x_{2}, y^{16}\right)$ are conjugate pairs of generators of $\mathbb{B}$, they are also conjugate in $\mathrm{GL}_{4370}(2)$. Hence a 'standard basis' algorithm as described in $[\mathbf{5}, \S 7.5 .3]$ will produce an element $g$ of $\mathrm{GL}_{4370}(2)$ which conjugates one pair to the other. Since the representation of $\mathbb{B}$ is irreducible, $g$ is unique up to scalar multiplication, and since the field has order 2, the element $g$ is actually unique. It follows that $g$ lies in $\mathbb{B}$.

\subsection{Step 3}

For Step 3, it was originally expected that we would use Ryba's algorithm [6] to achieve this. In fact, in the Baby Monster case we never tried to used Ryba's algorithm, and used the method described in $\S 3$, which was designed to give shorter words than Ryba's algorithm was likely to produce.

The best choice for $H_{1}$ in the Baby Monster is the largest subgroup, $2^{\cdot 2} \mathrm{E}_{6}(2): 2$, of index 13571955000 , and which can easily be found by the standard method of Bray [2]. Thus to 
perform the first step in our Baby Monster calculation, we expect (in the English sense) to create about 200000 cosets before finding the desired coincidence. This is an improvement on the naïve method by a factor of nearly $10^{5}$. Moreover, this subgroup fixes a unique non-zero vector in the given representation, so we can use vectors as labels for the cosets. (Even so, the storage requirement for 200000 vectors is at least $100 \mathrm{MB}$, even when stored in binary format.)

For the remaining steps of the calculation, both the groups and the representations are much smaller, and it is not necessary to take so much care over all the details. Our choice for the group $H_{2}$ was a group of shape $2 .\left(2_{+}^{1+20}: \mathrm{U}_{6}(2): 2\right)$, with index 3968055 in $H_{1}$. For this calculation, we chopped the representation (of $H_{1}$ ) to one of dimension 78 , such that there is an involution in the kernel of the (78-dimensional) representation. We adjust for this involution, if necessary, at the end of the whole calculation.

For $H_{3}$ we chose $2^{2} . \mathrm{U}_{6}(2) .2$, with index 1048576 in $H_{2}$, and order 73574645760 , so it is possible to choose $H_{4}$ to be the trivial group. Other chains of subgroups of $H_{3}$ are possible and may be easier to use. (Once we had found $H_{2}$ we worked in an even smaller representation that faithfully represented $2^{20}: \mathrm{U}_{6}(2): 2$, and so the effective index of $H_{4}$ in $H_{3}$ was $\left|\mathrm{U}_{6}(2): 2\right|=$ 18393661440$.

\section{References}

1. W. Bosma, J. Cannon and C. Playoust, 'The Magma algebra system. I. The user language. Computational algebra and number theory (London, 1993)', J. Symbolic Comput. 24 (1997) no. 3-4, 235-265.

2. J. N. BraY, 'An improved method for generating the centralizer of an involution', Arch. Math. (Basel) 74 (2000) 241-245.

3. J. J. CANnon et al., 'The Magma programming language'. Various versions, 1994-present.

4. J. H. Conway, R. T. Curtis, S. P. Norton, R. A. Parker and R. A. Wilson, An atlas of finite groups (Clarendon Press, Oxford, 1985).

5. D. F. Holt, B. Eick and E. A. O'Brien, Handbook of computational group theory. Discrete mathematics and its applications (Boca Raton) (Chapman \& Hall/CRC, Boca Raton, FL, 2005).

6. P. E. Holmes, S. A. Linton, E. A. O'Brien, A. J. E. Ryba and R. A. Wilson, 'Constructive membership in black-box groups', J. Group Theory 11 (2008) no. 6, 747-763.

7. S. A. Linton, 'The art and science of computing in large groups', Computational algebra and number theory (Sydney, 1992), Mathematics and Its Applications 325 (Kluwer Academic Publishers, Dordrecht, 1995) 91-109.

8. R. A. PARKer, 'The computer calculation of modular characters (the MeatAxe)', Computational group theory (Durham, 1982) (Academic Press, London, 1984) 267-274.

9. M. Ringe, 'The C-MeatAxe programming language', http://www.math.rwth-aachen.de/ MTX/.

10. R. A. Wilson, 'Some new subgroups of the Baby Monster', Bull. Lond. Math. Soc. 25 (1993) no. 1, $23-28$.

11. R. A. Wilson, R. A. Parker, S. J. Nickerson, J. N. Bray et al., 'Atlas of finite group representations, version 3' available at http://brauer.maths.qmul.ac.uk/Atlas/v3/.

John N. Bray

School of Mathematical Sciences

Queen Mary University of London

Mile End Road, London E1 4NS

United Kingdom

J.N.Bray@qmul.ac.uk

Robert A. Wilson

School of Mathematical Sciences

Queen Mary University of London

Mile End Road, London E1 4NS

United Kingdom

R.A.Wilson@qmul.ac.uk
Richard A. Parker

70 York Street

Cambridge CB1 2PY

United Kingdom

richpark54@hotmail.co.uk 